\title{
In vitro directed differentiation of mouse embryonic stem cells into insulin-producing cells
}

\author{
T. León-Quinto ${ }^{1}$ J. Jones ${ }^{1}$ A. Akoudy S M. Burcin $^{3} \cdot$ B. Soria ${ }^{1,4}$ \\ ${ }^{1}$ Institute of Bioengineering, School of Medicine, Miguel Hernández University, San Juan Campus, Alicante, Spain \\ 2 Cellular and Molecular Biology Unit, Municipal Institute of Medical Research (IMIM), Pompeu Fabra University, Barcelona \\ ${ }^{3}$ Cardion, Erkrath, Germany \\ ${ }^{4}$ Department of Surgery, National University of Singapore, Singapore
}

\section{Abstract}

Aims/hypothesis. We recently demonstrated that insulin-producing cells derived from embryonic stem cells normalise hyperglycaemia in transplanted diabetic mice. The differentiation and selection procedure, however, was successful in less than $5 \%$ of the assays performed. Thus, to improve its effectiveness, new strategies have been developed, which increase the number of islet cells or islet progenitors.

Methods. Mouse embryonic stem cells transfected with a plasmid containing the Nkx6.1 promoter gene followed by a neomycin-resistance gene, were cultured with factors known to participate in endocrine pancreatic development and factors that modulate signalling pathways involved in these processes. Neomycin was used to select the Nkx6.1-positive cells, which also express insulin. The transfected cells were differentiated using several exogenous agents, followed by selection of Nkx6.1-positive cells. The resulting cells were analysed for pancreatic gene and protein expression by im- munocytochemistry, RT-PCR and radioimmunoassay. Also, proliferation assays were performed, as well as transplantation to streptozotocin-induced diabetic mice. Results. The protocols yielded cell cultures with approximately $20 \%$ of cells co-expressing insulin and Pdx-1. Cell trapping selection yielded an almost pure population of insulin-positive cells, which expressed the beta cell genes/proteins Pdx-1, Nkx6.1, insulin, glucokinase, GLUT-2 and Sur-1. Subsequent transplantation to streptozotocin-induced diabetic mice normalised their glycaemia during the time period of experimentation, proving the efficiency of the protocols. Conclusions/interpretation. These methods were both highly efficient and very reproducible, resulting in a new strategy to obtain insulin-containing cells from stem cells with a near $100 \%$ success rate, while actively promoting the maturation of the exocytotic machinery.

Keywords Diabetes - Differentiation - Embryonic stem cells $\cdot$ Exogenous agents $\cdot$ Insulin-producing cells $\cdot$ Islet progenitors
Received: 3 February 2004 / Accepted: 19 April 2004

Published online: 29 July 2004

(C) Springer-Verlag 2004

\section{B. Soria (}

Institute of Bioengineering, School of Medicine,

Miguel Hernández University, San Juan Campus,

Carretera Alicante-Valencia Km 87, 03550 Alicante, Spain

E-mail: bernat.soria@umh.es

Tel.: +34-96-5919402, Fax: +34-96-5919539

Abbreviations: Anti-Shh, antibody against sonic hedgehog · D3, undifferentiated D3 stem cell line - EB, embryoid bodies · ES, embryonic stem $\cdot$ FBS, fetal bovine serum $\cdot$ LIF, leukaemia inhibitory factor $\cdot \mathrm{mES}$, mouse embryonic stem $\cdot \mathrm{Ngn} 3$, neurogenin $3 \cdot \mathrm{P}$, gelatine-coated plates $\cdot \mathrm{Pdx}-1$, pancreatic duodenum homeobox 1

\section{Introduction}

Islet transplantation may result in insulin independence for periods of 12 months or longer in patients with Type 1 diabetes [1]. However, this therapeutic approach will always be limited by the scarcity of human donors, so other alternative sources of beta cells have to be explored. Pluripotent stem cells may be a solution to this problem. We have recently shown that mouse embryonic stem (mES) cells can be driven to differentiate into insulin-secreting cells. Subsequent cell lineage selection with a cell trapping system based on the expression of a reporter-selector gene taking advantage of the insulin gene promoter allowed 


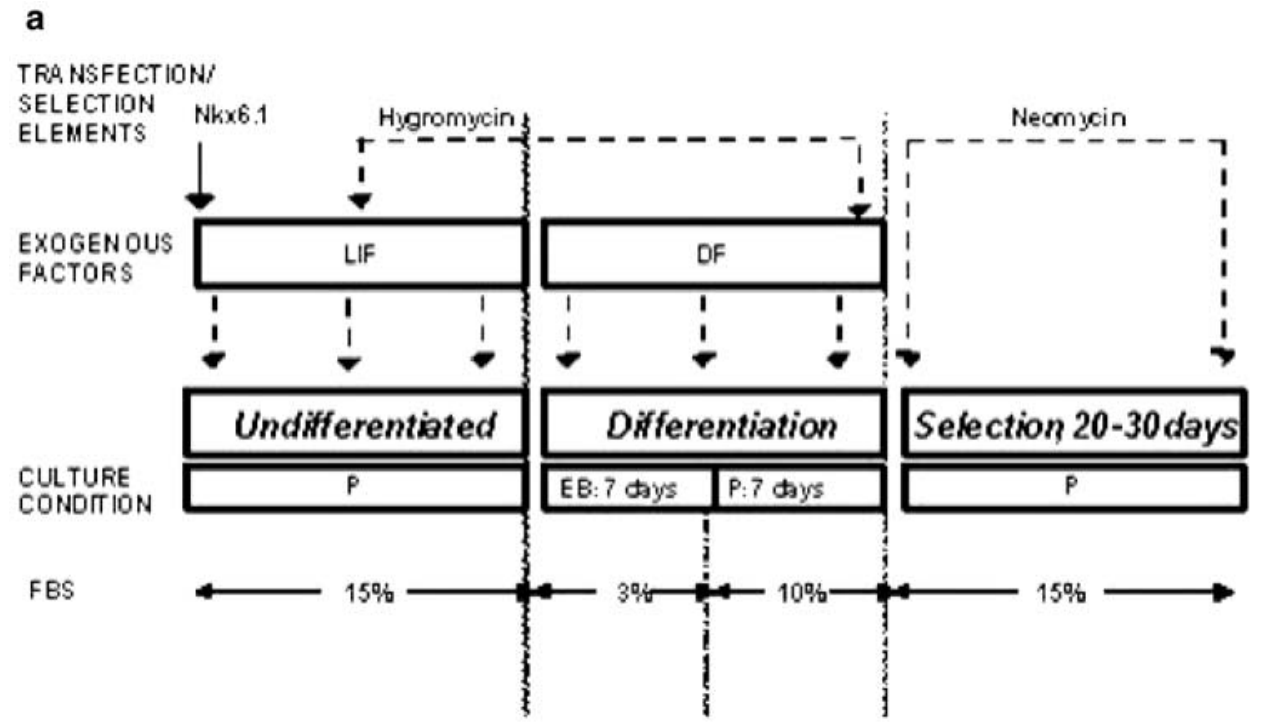

b

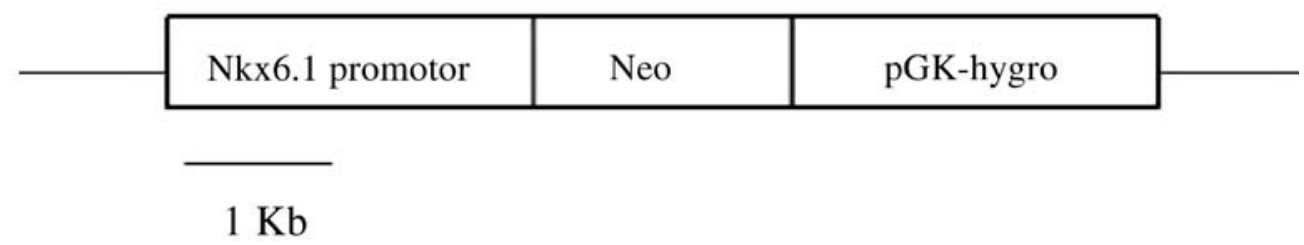

Fig. 1a. Differentiation-selection protocol. The differentiation-selection protocol consists of three steps: transfection of undifferentiated mES cells with a gene-trapping construct, directed differentiation into islet precursors, and neomycin selection of Nkx6.1-positive cells. b. Mouse Nkx6.1 promoter/pGKhygro $^{r}$ construction. DF, differentiating factors; EB, embryoid body phase; P, plating; Nkx6.1, selection construction

us to obtain insulin-producing cells that normalised hyperglycaemia when transplanted into the spleen of streptozotocin-induced diabetic mice [2]. Long-term follow-up of transplanted mice (42 weeks) showed that blood glucose levels remained normal and that insulin-secreting cells were present in the liver and in the spleen [3].

Generation of insulin-producing cells, however, was not very effective, with less than $5 \%$ of the assays being successful. A possible explanation for this low efficiency could be the low proportion of insulin-positive cells (less than 1\%) obtained during the first in vitro differentiation process, making cell selection more difficult. In addition, certain transcription factors involved in islet cell maturation and insulin expression, as well as beta cell products, are also expressed in other tissues. Such is the case for Pdx-1 expressed in the brain and insulin expressed in the retina [4]. Consequently, gene trapping strategies before differentiation may result in the selection of cells that express insulin but cannot be considered islet precursors. Thus, methods to increase the number of beta cell pre- cursors (cells positive for Pdx-1, Nkx6.1 and insulin) prior to cell lineage selection may be instrumental in driving pluripotent embryonic stem (ES) cells into insulin-containing cells with a precisely regulated insulin release, especially when applied to the differentiation of islet cells from pluripotent human ES cells [5].

Here, we describe a three-step differentiation-selection strategy based on current knowledge of transcription factors involved in beta cell development and differentiation, as well as of soluble factors or signalling pathways involved in the development of pancreatic beta cells (Fig. 1). For cell trapping, the Nkx6.1 gene promoter was used because this transcription factor is required for the expansion of beta cell precursors and is selectively expressed in beta cells in the adult pancreas $[6,7]$. It also appears in the enterochromaffin cells of the antropyloric mucosa of the stomach [8], but in this region there is no Pdx-1 or insulin expression, whereas in beta cells these three genes are expressed. To induce differentiation, in contrast with previous attempts [9], differentiation factors were added during the embryoid body formation. As a result, we obtained approximately $20 \%$ of the cells positive for insulin and Pdx-1, which amounted to almost $100 \%$ insulin-positive cells after cell lineage selection with the Nkx6.1-neo plasmid. When these cells were transplanted under the kidney capsule of diabetic mice, their glycaemia was normalised and body weight recovered, proving the efficiency of the protocol. 


\section{Materials and methods}

Culture of mES cells. D3 mouse cell line was cultured on gelatine-coated flasks with high-glucose DMEM (Gibco/BRL, Grand Island, N.Y., USA) containing $15 \%$ fetal bovine serum (FBS; Hyclone, Logan, Utah, USA), 1\% non-essential amino acids (Gibco/BRL), $0.1 \mathrm{mmol} / \mathrm{l}$ 2-mercaptoethanol (Gibco/ BRL), $1 \mathrm{mmol} / 1$ sodium pyruvate, $1000 \mathrm{U} / \mathrm{ml}$ mouse recombinant leukaemia inhibitory factor (LIF; Gibco/BRL), $100 \mathrm{U} / \mathrm{ml}$ penicillin and $0.1 \mathrm{mg} / \mathrm{ml}$ streptomycin.

Transfection of mES cells with Nkx6.1-neo plasmid. Undifferentiated D3 cells were transfected by electroporation with a mouse $N k x 6.1$ promoter/ $p G K$-hygror construction. A $2.4-\mathrm{Kb}$ fragment of the Nkx6.1 promoter was amplified from mouse genomic DNA by PCR using the following primer: Nkx up- $5^{\prime}$ GACATTCAGAGTCAAACTCCCTGGC 3'; Nkx low-5' CCCAAGCCAGACCCGAACC 3'. The amplified product was cloned into a pcr2.1 vector that generated the construct $N k x 6.1$ pcr2.1. After verification by sequencing, the promoter fragment was again excised with BamH1/EcoRV and cloned into the vector neoIRES LacZ pGK hygro to generate the final construct pRIPneo-IRES-LacZ-hygro.

This way the $N k x 6.1$ promoter is placed above the neomycin resistance gene, thus permitting neomycin resistance when Nkx6.1 is activated. The constitutive-expression-driven hygromycin resistance gene placed on the same construct allows selection of transfected transgenic cells, harbouring at least one copy of the Nkx6.1 construct. The $7.9-\mathrm{Kb}$ plasmid was linearised with BamHI digestion and transfected in D3 ES cells by electroporation. After 2 days, transfected cells were cultured in our standard medium and selected by growth with $800 \mu \mathrm{g} / \mathrm{ml}$ hygromycin (Calbiochem, La Jolla, Calif., USA).

Differentiation-selection protocol. To direct the differentiation, besides eliminating LIF from the medium, we combined the reduction in serum concentration (from $15 \%$ to $3 \%$ ) with the addition of selected factors. The factors used were $2 \mu \mathrm{g} / \mathrm{ml}$ anti-sonic hedgehog (Developmental Studies Hybridoma Bank, Iowa City, Iowa, USA) and $10 \mathrm{mmol} / \mathrm{l}$ nicotinamide (Sigma-Aldrich) [10]. In vivo, sonic hedgehog (Shh) is inhibited in the developing pancreas of embryonic mice, permitting the expression of Pdx-1 and the subsequent cascade of pancreatic genes. Thus, we use an antibody that specifically blocks the Shh receptor Patched.

In addition, cells were co-cultured with embryonic pancreases from embryonic day 17.5 (e17.5), in which the soluble factors liberated by the forming islets induce differentiation. Embryos from time-mated pregnant OF1 mice (e17.5; Charles River Laboratories, France) were liberated in Hank's balanced salt solution (Gibco/BRL). Whole pancreases were collected by microdissection, washed in Hank's solution several times and transferred to Petri dishes, where D3 cells were grown. Pancreatic rudiments of seven to eight fetuses were plated on each Petri dish. Pancreatic rudiments and mES cells were separated by nylon filters with a pore size of $0.2 \mu \mathrm{m}$ (Millipore, Bedford, Mass., USA). The pancreatic rudiments were placed in the bottom of the plate, then the nylon filter was placed, and the embryoid bodies placed on top of the filter. This ensures that the ES cells and the pancreatic rudiments are never in physical contact. We explored the possibility that cells from the pancreatic rudiments might pass to the embryoid bodies region, thereby contaminating it, but simultaneous assays where embryoid bodies were grown in separate plates with medium derived from pancreatic rudiments show similar results. Also, neomycin included in the selection phase would destroy all cells that do not contain the plasmid, including pancreatic contaminants.

To begin the differentiation, 5 million cells were cultured for 7 days to form embryoid bodies (EB, 3\% FBS) and plated for 7 days (P; 10\% FBS). During this 14-day period, the cells were treated with the above-mentioned factors, except in the case of anti-sonic hedgehog and co-culture with pancreatic rudiments, which were present only during the 7 days of EB formation.

Afterwards, the differentiated cultures were grown in D3 cell culture medium, without LIF and penicillin/streptomycin,

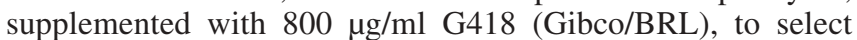
Nkx6.1-positive cells. These cells were cultured for an additional 20 to 30 days.

Cell proliferation analysis and BrdU incorporation. After trypsinisation of undifferentiated stem cells, cells were counted and cultured using the differentiation protocols previously described. After 7 days of EB formation and 7 days of plating, the plates were trypsinised and counted, so that the number of population doublings could be determined. Using neomycinselected cells, cell count was done approximately 2 days after neomycin was added, when only Nkx6.1-positive cells survived, and then counted again 20 days later, to calculate the number of population doublings.

For BrdU staining, the cells were incubated with $10 \mu \mathrm{mol} / 1$ BrdU (Sigma) for $24 \mathrm{~h}$. Then, the cells were fixed with $4 \%$ paraformaldehyde, and DNA was denatured using $4 \mathrm{~mol} / \mathrm{l} \mathrm{HCl}$. The rest of the protocol is a standard immunocytochemistry protocol, described below. Mouse anti-BrdU antibody 1:1000 (Sigma) was used as the primary antibody, and anti-mouse FITC 1:300 (Sigma) was used as the secondary antibody.

RT-PCR analysis. RNA was isolated from differentiated ES cells as described by Chomczynski [11]. Total RNA $(1 \mu \mathrm{g})$ was reverse-transcribed and cDNAs were amplified using the $\mathrm{Su}$ perscript one-step RT-PCR kit (Gibco). Reactions (25 $\mu \mathrm{l})$ containing mRNA-specific primers (5 pmol each) were incubated at optimal annealing temperatures (as described below) and subjected to 30, 35 and 40 cycles of amplification unless otherwise indicated. The PCR products were separated using $2 \%$ agarose gels and stained with ethidium bromide. The oligonucleotide pairs used for PCR and the size of the amplified products were as follows: Nkx6.1 (55 $\left.{ }^{\circ} \mathrm{C}\right)$ TCTTCTGGCCNGGGGTGATG (S) and CCTGCTTCTTCTTGGCGGTG (AS) (311 bp); Sur-1 $\left(60{ }^{\circ} \mathrm{C}\right)$ GATGCCATCATCACAGAAGG (S) and ATCACGAGGTCTGCACTCAG (AS) (236 bp); glucokinase $\left(62{ }^{\circ} \mathrm{C}\right)$ AGATCCTGGCAGAGTTCCAG (S) and CTTTCACCAGCATCACCCTG (AS) (219 bp); GLUT-2 $\left(62{ }^{\circ} \mathrm{C}\right)$ ACACCCCACTCATAGTCACAC $(\mathrm{S})$ and CAGCAATGATGAGAGCATGTG (AS) (269 bp); insulin [12]; Pdx-1 and NeuroD [13]; ngn3 [14]; and $\beta$-actin [15]. All experiments were carried out in duplicate and the reproducibility of the observations was confirmed in three independent experiments. RNA expression was estimated based on the intensity of the bands of the PCR products as compared with the intensity of the band corresponding to $\beta$-actin.

Immunocytochemistry. A standard immunocytochemistry protocol was used. Cells were fixed with $4 \%$ paraformaldehyde and permeabilised with $0.1 \%$ Triton $\mathrm{X}-100$. The following primary antibodies were used: mouse anti-insulin (1:1000; Sigma-Aldrich), rabbit anti-Nkx6.1 (1:4000; kindly provided by O. Madsen) and rabbit anti-Pdx-1. The rabbit anti-Pdx-1 (1:20) was developed in our laboratory as previously described [16] using the peptide sequence published elsewhere [17]. AntiPdx-1 antibody is polyclonal, binding to both nucleus and cy- 
toplasmic (phosphorylated) isoforms, its efficiency proven in another article [18]. Primary antibody localisation was done using anti-mouse FITC (1:300; Sigma-Aldrich) for insulin, and anti-rabbit FITC (1:300; Sigma-Aldrich) for Pdx-1 and Nkx6.1. Proper controls for secondary antibodies revealed no non-specific staining. Cells were counter-stained with $1 \mu \mathrm{mol} / \mathrm{l}$ homodimer ethidium (Molecular Probes, Leiden, The Netherlands) for $1 \mathrm{~min}$ before visualisation under a Zeiss LSM 510 confocal microscope (40× objective, $1.3 \mathrm{NA})$.

Insulin content and secretion assays. To determine total insulin content, 250,000 to $1,000,000$ cells were incubated for $24 \mathrm{~h}$ with ethanol/ $\mathrm{HCl}$ buffer at $4{ }^{\circ} \mathrm{C}$. Cellular extract was determined for insulin. For insulin secretion, cells were plated in 12 -well dishes at a density of 100,000 to 250,000 cells per well and allowed to grow overnight in the same medium as the neomycin-selected cells. Cells were then washed twice for $10 \mathrm{~min}$ each in fresh phosphate buffer solution (Invitrogen) and incubated for $4 \mathrm{~h}$ in $0.5 \mathrm{ml}$ of fresh modified Krebs buffer with $3 \mathrm{mmol} / \mathrm{l}$ glucose. Afterwards, cells were incubated $\left(95 \% \mathrm{O}_{2}\right.$, $5 \% \mathrm{CO}_{2}$ ) at $37{ }^{\circ} \mathrm{C}$ for $1 \mathrm{~h}$ in $0.25 \mathrm{ml}$ of the fresh modified Krebs buffer with $3 \mathrm{mmol} / \mathrm{l}$ glucose, followed by a 1-h incubation period with $22 \mathrm{mmol} / \mathrm{l}$ glucose. Mouse islets were used as positive controls (from 5 islets to 50), obtained from 2-monthold OF1 mice. At the end of the incubation period, the buffer was removed and studied for insulin content. Insulin was determined by RIA using the Coat-a-Count kit (DPC, Los Angeles, Calif., USA).

Induction of hyperglycaemia by streptozotocin and treatment with neomycin-selected cells. OF1 male mice of 2 to 3 months old (Charles River Laboratories, France) were treated with $200 \mathrm{mg} / \mathrm{kg}$ streptozotocin in citrate buffer. Then, 5 to 7 days later, mice were injected with 4.5 million neomycin-selected cells derived from the pancreatic rudiments protocol, in the suprarenal gland of the kidney. Treated mice were maintained for 3 weeks, after which the graft was removed. Glucose levels were determined in blood samples drawn from the tail vein. A week after graft removal, the mice were killed by decapitation under anaesthesia and the pancreas was removed for study.

The graft and pancreas of the mice were treated with a gradient of saccharose, and 8-mm cryosections were obtained for immunohistochemistry. The immunohistochemistry protocol was identical to the immunocytochemistry protocol, using Nkx6.1 and insulin antibodies, along with nuclear staining with homodimer ethidium. All procedures were approved by the local ethics commitee and the institutional review board.

\section{Results}

mES cells differentiate towards insulin- and Pdx-1positive cells. The three-step differentiation-selection protocol (Fig. 1a) consists of (i) transfection of undifferentiated mES cells with a gene-trapping construct, (ii) directed differentiation into islet precursors, and (iii) neomycin selection of $N k x 6.1$-expressing cells to enrich the insulin-producing cell population. In our work, we used the $N k x 6.1$ promoter gene to drive selection towards differentiated insulin-producing cells (Fig. 1b). This gene appears initially in almost all of the epithelial cells of the pancreatic buds, until around embryonic day 13 (e13), where its expression becomes restricted to in beta cells [19]. Even though this transcription factor may appear in other tissues, such as the stomach [8], we believe that Nkx6.1 would be a very good candidate gene for our construct, provided that cells are selected after directed differentiation. We also included a hygromycin resistance gene in the plasmid, which is expressed continuously in the cell by a pGK promoter. In this manner, when hygromycin is included in the media, only the cells containing the plasmid survive. Hygromycin is included in the first two steps of the differentiation-selection protocol, but not in the neomycin selection phase.

The next step involved directing the differentiation of mES cells using exogenous factors. We tested a long list of factors (LY294002, retinoic acid, sodium tungstate, cyclopamine, nicotinamide, co-culture with pancreatic rudiments of different embryonic days etc.) and eliminated those that gave poor results in terms of Nkx6.1 expression. The factors resulting in the highest Nkx6.1 expression were nicotinamide, antibodies against Shh, and the co-culture with pancreatic rudiments of e17.5 fetal mice. The effectiveness of nicotinamide in stem cell differentiation has already been proven [2]. Anti-Shh inhibits sonic hedgehog, thus permitting Pdx-1 expression. And, as for the co-culture of pancreatic rudiments at e17.5, the soluble factors released by the rudiments may direct the differentiation of stem cells towards the pancreatic destination.

Gene expression of four early transcription factors (Pdx-1, Nkx6.1, NeuroD and Ngn3) as well as insulin expression of pre-selected cells is shown in Figure 2a. At low cycles, RT-PCR is a semiquantitative approach to gene expression. In this manner, $\mathrm{Pdx}-1$ gene expression increased in the cells co-cultured with pancreatic rudiments, and to a lesser extent, in the anti-Shh and the control protocols. NeuroD and Neurogenin 3 transcripts were detected at low levels in almost all of the protocols analysed, except for anti-Shh which induced a notable increase in expression. Nkx6.1 expression occurred in all of the protocols to a greater or lesser extent. The highest levels of insulin mRNA expression were obtained using co-culture with pancreatic rudiments and anti-Shh protocols. At lower cycles, insulin mRNA expression was only detected in the pancreatic rudiment protocol (data not shown).

With respect to $N k x 6.1$-selected cells (Fig. 2b), all protocols seemed to have similar gene expression levels of Pdx-1, Nkx6.1, insulin, glucokinase, GLUT-2 and Sur-1 (all at 35 cycles). Only in the case of insulin expression was there a notable difference, where coculture with pancreatic rudiments seemed to express more than the other protocols.

Thus, analysis by RT-PCR demonstrates that using various factors during differentiation increases the expression of pancreatic genes to different extents before selection is initiated. The most successful factors have been pancreatic rudiments and anti-Shh, yielding the most remarkable increases of all the endocrine tran- 


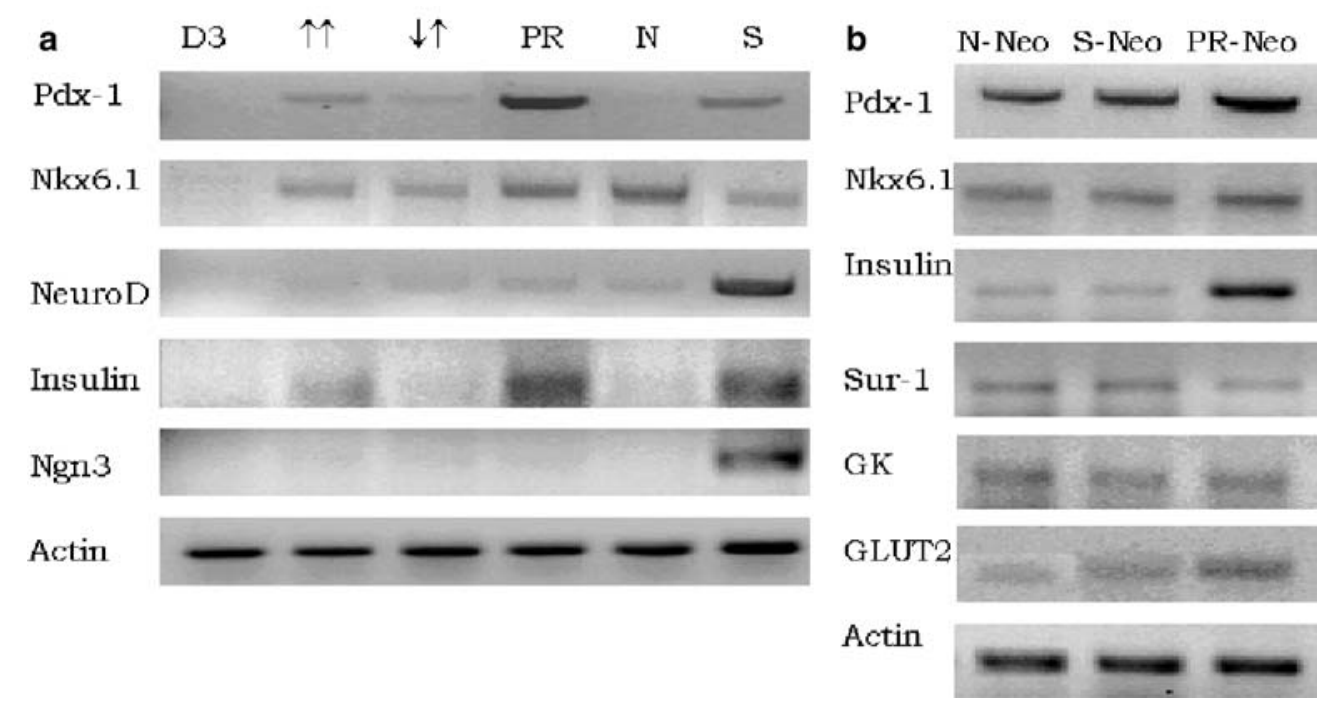

Fig. 2. RT-PCR analysis of islet precursor markers in in vitro differentiated mES cells, pre-selection (a) and post-selection (b) (35 cycles). The columns represent protocols used. Lines are the islet precursor markers analysed. $\uparrow \uparrow$, control: 7 days embryoid body formation (EB; $15 \%$ serum) followed by 7 days in gelatin-coated plates (P; 15\% serum); $\downarrow \uparrow$, control: 7 days EB (3\% serum) followed by 7 days P (10\% serum); D3, undifferentiated cells; PR, pancreatic rudiments; $\mathrm{N}$, nicotinamide; S, anti-sonic hedgehog; Neo, neomycin-selected protocols (neomycin cell selection after application of the corresponding protocol); GK, glucokinase

scription factors and insulin transcripts analysed before selection. After selection, expression levels seemed to be similar in all protocols (Fig. 2), proving that cells expressing islet transcription factors were selected.

The effects of the agents were also analysed by immunocytochemistry using antibodies detecting Pdx-1, Nkx6.1 and insulin (Fig. 3). Without selection, under low FBS culture conditions, the proportion of cells expressing Pdx-1, Nkx6.1 and insulin was always under 10\% (Fig. 3a). Co-culture with pancreatic rudiments drove $32 \pm 2 \%(n=8)$ of the cells to express Pdx-1, and $19 \pm 1 \%(n=8)$ to co-express Pdx-1 and insulin. With respect to Nkx6.1, the most effective factors were nicotinamide $(20 \pm 1 \%, n=3)$ and co-culture with pancreatic rudiments $(16 \pm 5 \%, n=5)$. After neomycin selection, nearly all cells stained for Pdx-1 and Nkx6.1, and nearly all were insulin positive (Fig. 3b-d).

Cell proliferation varies in the different protocols. Figure 4 depicts the population doublings (Fig. 4a) and BrdU incorporation (Fig. 4b) of the resulting cells cultured from the various protocols used, before and after neomycin selection. Undifferentiated D3 cells duplicated their population approximately ten times in 14 days, and nearly $100 \%$ of the cells were BrdU-positive after $24 \mathrm{~h}$ of incubation. Compared with these cells, all differentiation protocols, including the controls, showed a remarkable decrease in cell growth.
Both controls and anti-Shh protocols showed similar population doublings. Nicotinamide and pancreatic rudiments protocols reduced cell proliferation significantly, in a very similar manner. Thus, cell proliferation analysis before selection showed that all the protocols, including the controls, greatly decrease cell proliferation compared with undifferentiated cells, in favour of differentiation.

After neomycin selection, the proliferation rate decreased in all of the protocols. If the data shown in Figure $4 \mathrm{a}$ were represented as population doublings per day, the differentiated cells before neomycin selection would duplicate about 0.22 to 0.30 per day, whereas the neomycin-selected cells would be in the 0.05 to 0.18 doublings per day range, resulting in a 40 to $80 \%$ decrease in proliferation. These data indicate that cells selected with neomycin are less proliferative, in favour of differentiation, than the rest of the cells in culture before the selection. At this stage cell proliferation must be as low as possible, because, if cells are used in cell transplantation, a high proliferative value may indicate a greater possibility of tumour formation.

One of the most obvious differences among the various protocols is the cell survival rate immediately after neomycin selection. In the case of the control protocols, there was massive cell death 2 days after neomycin was added to the medium, with only $0.01 \%$ to $0.02 \%$ of the cells surviving (one in 5000 to 10,000 cells; data not shown). In the case of the other protocols, $15 \%$ of the cells survived with nicotinamide treatment, $10 \%$ survived in the pancreatic rudiment protocol, and 5\% survived with anti-Shh treatment. These values are similar to the percentages of Nkx6.1positive cells seen in immunocytochemistry (Fig. 3a) and are 500 to 1500 times as great as in the controls. It is very important to analyse the cell survival rate of the cells after neomycin selection to know which protocol is best for use with the Nkx6.1 plasmid. 
a
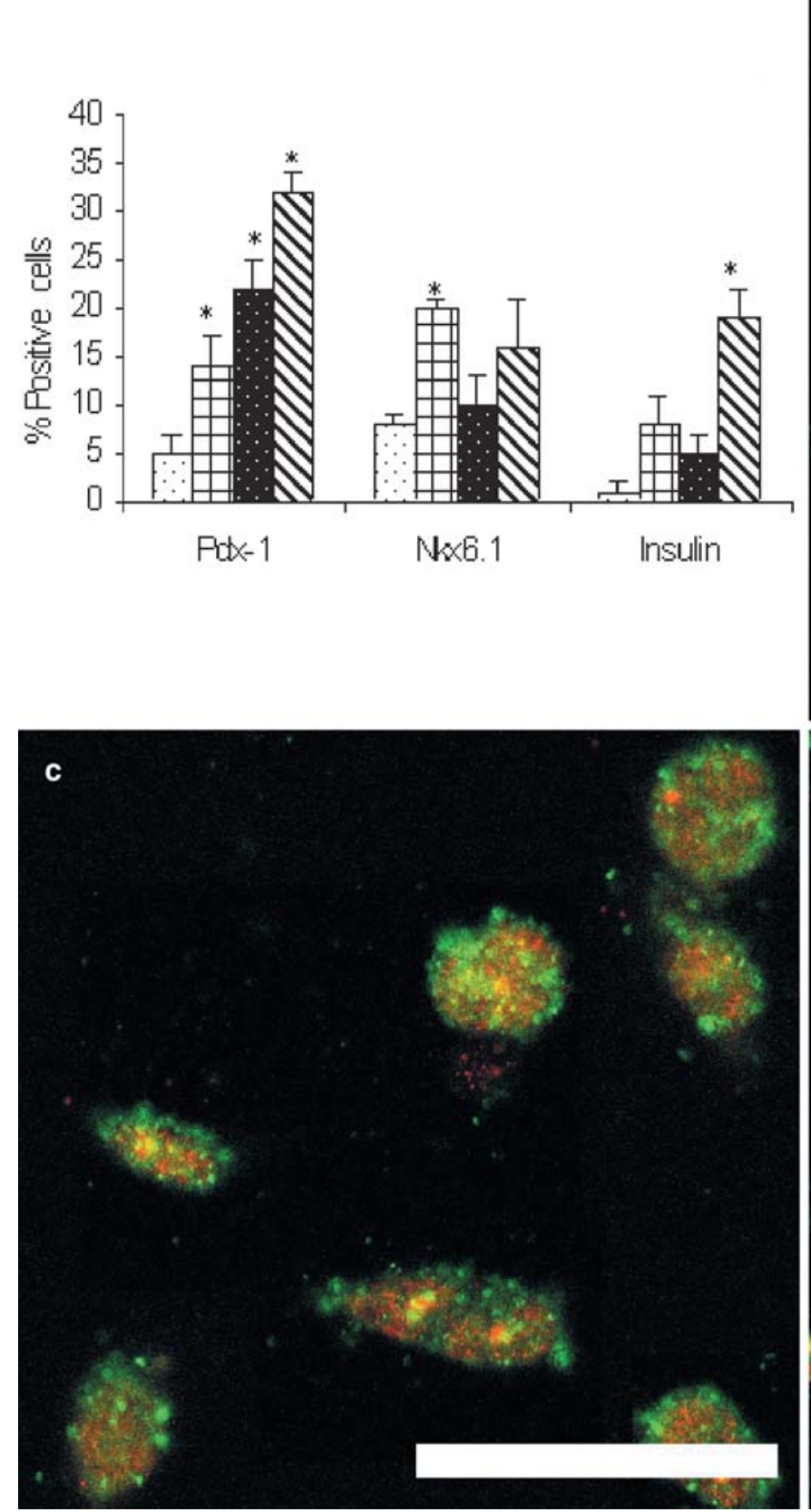

Fig. 3. Immunocytochemical analysis of cells submitted to the indicated differentiation protocols. a. Histogram representing the percentage of pre-selected cells expressing Pdx-1, Nkx6.1 and insulin. The columns for each protein analysed are (from left to right): control 3\%/10\%, nicotinamide, anti-Shh and pancreatic rudiments. Neomycin-selected cells, regardless of protocol, were nearly $100 \%$ positive for Pdx-1, Nkx6.1 and insulin. ${ }^{*} p<0.05$ compared with control $3 \% / 10 \%$. b. Pdx-1-positive (red) and insulin-positive (green) cells. c. Nkx6.1-positive (red) and insulin-positive (green) cells. d. Insulin staining (green) and nuclear staining (red), presenting nearly $100 \%$ insulin-positive cells. The immunocytochemistry images shown represent cells that have been cultured with anti-Shh and selected with neomycin for 25 days. Bar $=50 \mu \mathrm{m}$
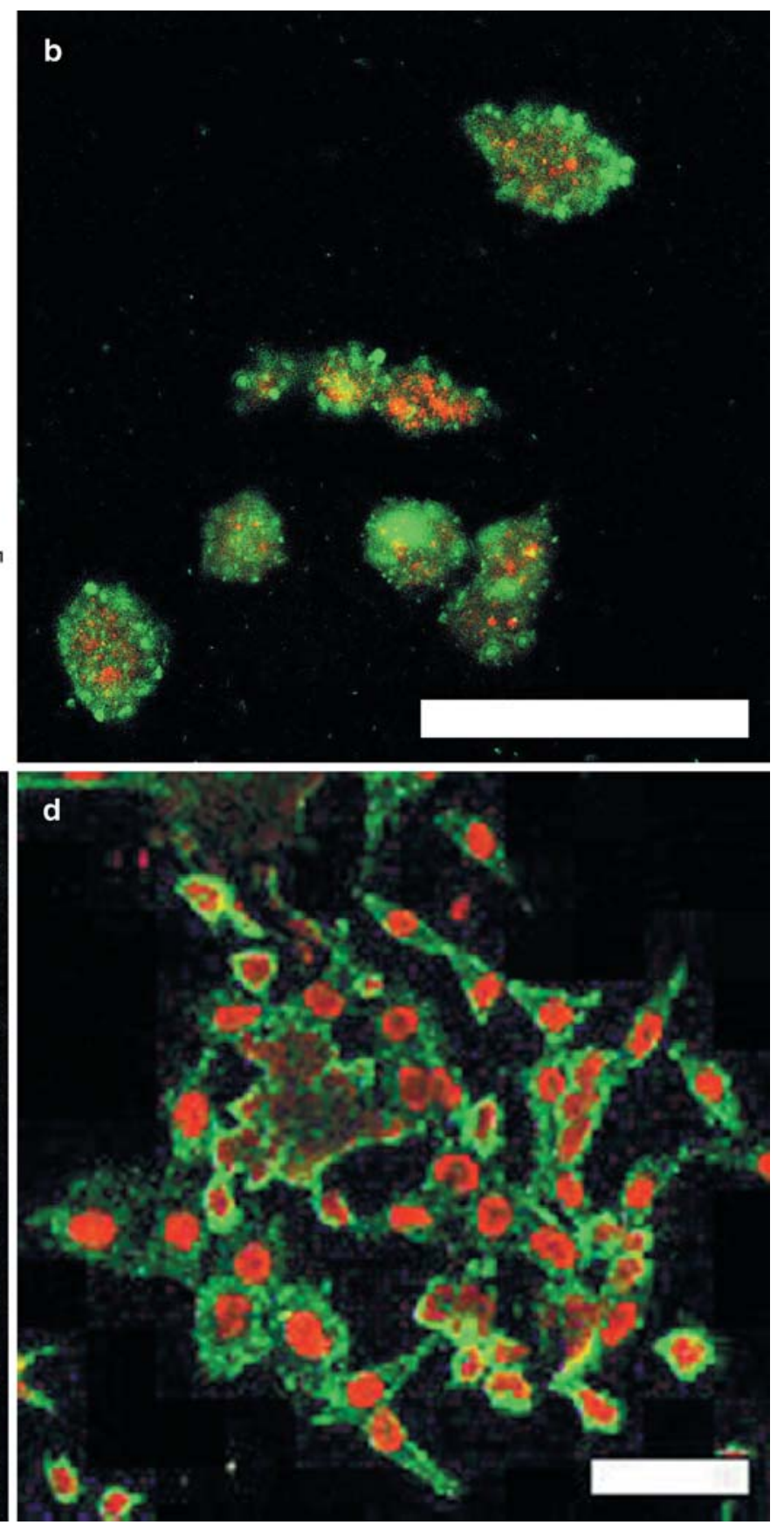

Insulin content and release assay. Neomycin-selected cells of all the protocols were analysed for insulin content and secretion. Without neomycin selection, insulin content was very low in all the protocols (2-3 ng/mg protein; data not shown), yet after selection, this value increased 100 -fold, to between 150 and $200 \mathrm{ng} / \mathrm{mg}$ protein (Fig. 5, Table 1). Insulin content values were similar among the various protocols. The main difference between the protocols was in the standard deviations, which varied greatly among the controls, as opposed to in the agents, which had low standard deviations. This indicates that in the protocols with no factors added (considered the controls) and in which selection was done, the selected cells were in various degrees of maturation, while the cells 


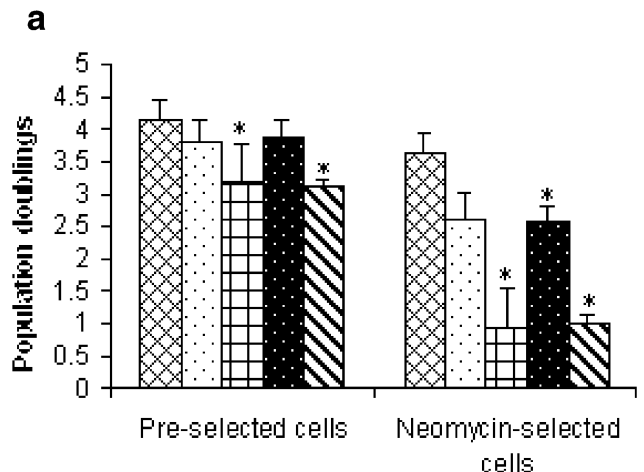

b

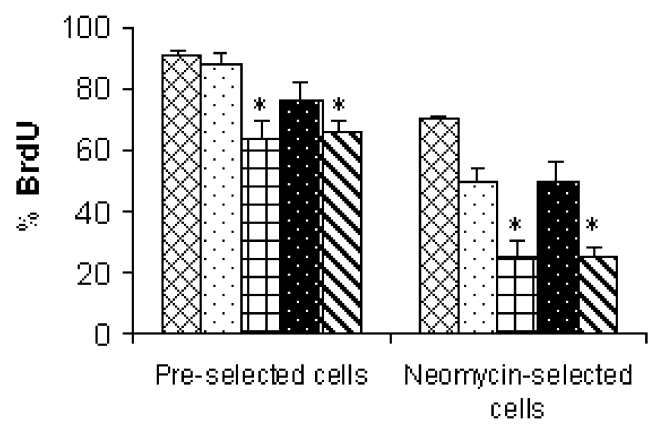

Fig. 4. Cell proliferation analysis. a. Population doublings. The $y$ axis indicates the number of population doublings for each of the protocols analysed, in the protocols before and after neomycin treatment. In the case of pre-selected cells, the histogram indicates the number of population doublings in 14 days, whereas in the case of neomycin-selected cells, the number of population doublings in 20 days of culture is indicated. b. BrdU incorporation of the various protocols. All differentiation protocols show a significant decrease in the number of BrdU-positive cells after neomycin treatment, which is also reflected in the population doublings seen in $\mathbf{a}$. In both a and $\mathbf{b}$, the columns represent (from left to right): control $15 \%$, control 3\%/10\%, nicotinamide, anti-Shh and pancreatic rudiments. Control 15\%, control with EB formation for 7 days followed by 7 days of plating, all under $15 \%$ serum; control $3 \% / 10 \%$, control with EB formation for 7 days (3\% serum) followed by 7 days of plating ( $10 \%$ serum). $n=6$ in all cases; $* p<0.05$ compared with control $15 \%$

differentiated by the agents all seemed to be in a more mature state.

Analysing insulin secretion, however, gave an interesting result. In both controls, no insulin secretion was detected in $22 \mathrm{mmol} / \mathrm{l}$ glucose media, demonstrating a deficient signalling transduction pathway for glucose responsiveness in these cells, probably caused by a state of lesser maturation. The other factor-driven protocols showed glucose-stimulated insulin release, incrementing the insulin in the media five to twelve times. Comparing these data with the insulin content, it can be seen that 2 to $3 \%$ of the total insulin content was released, similar to in beta cells. Thus, these cells seem to possess all of the mechanisms necessary to release insulin in a glucose-responsive manner.

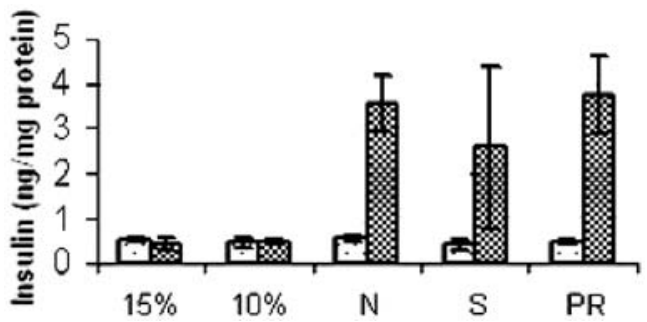

Fig. 5. Insulin content and secretion. In the case of insulin secretion, the insulin content in the media at $3 \mathrm{mmol} / \mathrm{l}$ glucose was, on average, $0.44 \pm 0.15 \mathrm{ng} / \mathrm{mg}$ protein. Insulin release was 2 to $3 \%$ of the total insulin content, incrementing the insulin in the media five to twelve times compared with the $3 \mathrm{mmol} / \mathrm{l}$ glucose media ( $n=3$ in all cases except for pancreatic islets, in which case $n=6$ ). $\mathrm{N}$, nicotinamide; S, anti-sonic hedgehog; PR, pancreatic rudiments

Table 1.

\begin{tabular}{lc}
\hline $\begin{array}{l}\text { Protocol } \\
(20-30 \text { days neomycin) }\end{array}$ & $\begin{array}{l}\text { Insulin content } \\
\text { (ng/mg protein) }\end{array}$ \\
\hline Control 15\% & $210 \pm 120$ \\
Control 3\%/10\% & $210 \pm 110$ \\
Nicotinamide & $160 \pm 40$ \\
Anti-Shh & $160 \pm 20$ \\
Pancreatic rudiments & $160 \pm 40$ \\
Pancreatic islets & $20,310 \pm 4570$ \\
\hline
\end{tabular}

The left column represents the insulin level at $3 \mathrm{mmol} / \mathrm{lglu}$ cose for each protocol; the right column is the insulin level at $22 \mathrm{mmol} / \mathrm{l}$

Normalisation of glycaemia in diabetic mice after transplantation of ES-derived insulin-producing cells. A week before the transplantation, the mice were injected with $200 \mathrm{mg} / \mathrm{kg}$ streptozotocin. Half or more than half of the mice died during the week that followed due to very rapid and high glycaemia rates $(>16.7 \mathrm{mmol} / \mathrm{l})$. In the surviving mice, glycaemia was slightly higher 2 days after the injection, on average $8.3 \mathrm{mmol} / \mathrm{l}$ (Fig. 6a). However, the glycaemia increased until the animals had an average of $17.8 \mathrm{mmol} / \mathrm{l}$ glucose at the day of transplantation. Injected into the kidney capsule of the diabetic animals were 4.5 million insulin-producing cells derived from the pancreatic rudiment protocol. After transplantation, the glycaemia steadily decreased down to about $4.7 \mathrm{mmol} / \mathrm{l}$, then increased again to $8.3 \mathrm{mmol} / \mathrm{l}$ (non-diabetic mice maintain a glycaemia of $4.4-6.7 \mathrm{mmol} / \mathrm{l})$. This glucose level was maintained throughout the whole experiment for a total of 3 weeks, after which the transplant was removed to confirm that the cells transplanted were the cells responsible for the glucose normalisation. No tumour formation was observed when the graft was removed. Finally, a week after graft removal, the mice were killed and the pancreas was obtained. Undifferentiated ES cells were transplanted in 

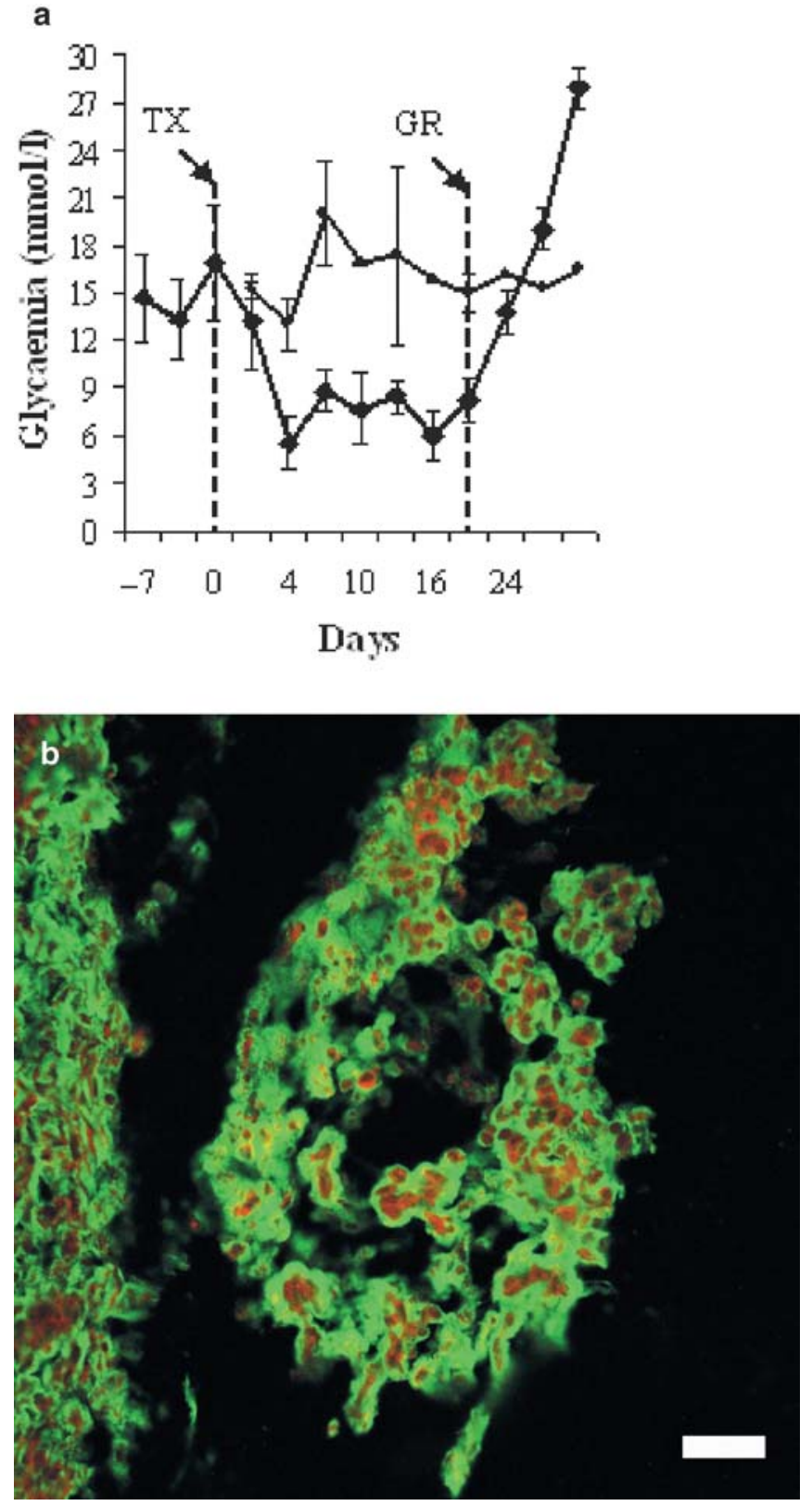

Fig. 6. Blood glucose normoglycaemia after transplantation of insulin-producing cells into streptozotocin-induced diabetic mice. a. The values between the two vertical dotted lines are the glycaemia of the mice with the insulin-producing cells $(n=4)$. Dotted line indicates diabetic mice that have not been implanted with the cells $(n=3)$. b. Nkx6.1 and insulin immunostaining in the kidney graft. Red indicates Nkx6.1, green indicates insulin. TX, transplantation; GR, graft removal. Bar = $50 \mu \mathrm{m}$

diabetic mice, which did not induce normoglycaemia, and provoked teratoma formation (data not shown).

Both tissues, the graft and pancreas, were treated with saccharose, and 8-mm thick tissue samples were obtained for immunohistochemistry. The graft presented cells that were positive for Nkx6.1 and insulin (Fig. 6b), whereas the pancreas was negative for both. Thus, glucose normalisation was obtained using the transplanted cells, no tumour formation was observed, and the pancreas did not present insulin-positive cells.

\section{Discussion}

In our previous work [2], we used cell lineage selection in a similar manner as shown here. However, the population of cells expressing insulin in the selection process was less than $1 \%$. Also, this strategy was not very efficient, with less than $5 \%$ of the assays performed being successful. Thus, in this paper we present a new process of differentiation where we end up with insulin-producing cells in all the assays performed. To be certain that the cells obtained are of pancreatic origin, a panel of pancreatic genes, such as Pdx-1, Nkx6.1, NeuroD and Neurogenin 3, needs to be analysed.

The aim of this study was to optimise strategies to engineer islet cells from stem cells. As a result, we were able to obtain cell cultures where up to $20 \%$ of cells co-expressed insulin and Pdx-1. After neomycin selection, we obtained cell cultures where almost $100 \%$ of the cells were positive for beta-cell-specific genes and proteins ( $P d x-1$, insulin, Nkx6.1 etc.). Cell survival varied among the protocols, showing survival rates 500 to 1500 times higher with nicotinamide, anti-Shh or pancreatic rudiments than with the controls. This shows that the factors analysed in this study are effective using the described differentiation-selection protocol. Analysis of insulin content and release revealed that under control conditions (low FCS, embryoid body formation), cells may undergo random differentiation with a high variability in insulin content and lack of regulated insulin release. Only the cells exposed to differentiation factors (nicotinamide, anti-Shh, soluble factors from pancreatic rudiments) mature their secretory machinery to respond to glucose in a regulated form. Among the agents analysed, co-culture with pancreatic rudiments and exposure to anti-Shh antibodies have been effective methods to increase the number of Pdx-1-positive islet progenitor cells, whilst nicotinamide is more effective in promoting the appearance of Nkx6.1-positive cells. This was shown by RT-PCR and immunocytochemistry. While the insulin content is similar to in other differentiation protocols [20], these protocols differ by generating cells with a higher state of maturation, shown by the glucose-dependent insulin release of the generated cells. Depending on the proto$\mathrm{col}$, the concentration of insulin in the media increased five- to twelve-fold when $22 \mathrm{mmol} / \mathrm{l}$ glucose was added.

In addition to the lack of regulated insulin release, control protocols also displayed low cell survival, making them non-efficient for use in clinical applications. In order to develop a protocol that ensures a 
high enough cell mass for cellular therapy against diabetes, cell proliferation analysis was performed to identify a factor that results in a high percentage of insulin-positive cells after antibiotic selection. Cells differentiated without any agents were slightly less proliferative after neomycin selection, as compared with the cells cultivated together with the agents that greatly affected proliferation.

Transplantation of the selected cells into diabetic mice resulted in glucose normalisation. Blood glucose was normalised for more than a month without evidence of tumour formation. Mice transplanted with undifferentiated D3 cells develop teratomas in periods of 3 to 4 weeks. To discard any contribution due to islet regeneration, the graft was removed 3 weeks after transplantation, which resulted in a progressive increase in blood glucose levels in the week following the operation. Immunohistochemistry analysis demonstrated that the pancreas did not present insulin or Nkx6.1-positive cells, as opposed to the graft. Thus, the transplanted cells were mature enough to respond to the high glucose levels of the mice and regulate its glycaemia during the weeks that followed the transplantation, with no indication of tumour formation or pancreas regeneration.

A practicality to consider is that when these cells have gone through selection and are frozen in liquid nitrogen and then re-thawed, their insulin content and secretion is greatly affected. The data shown here have always been obtained with cells that have never been frozen and thawed. Little or no insulin content or secretion was detected in frozen cells. Thus, the freezing and thawing process affects the cells to the point of them not regaining their function even after several days or weeks of culture. Trypsinisation also affects neomycin-selected cells, resulting in proliferation of the cells to the same extent as cells without selection. We do not currently have an explanation for these findings, but they may indicate de-differentiation of the cells and possible potential for tumour formation if used in cell therapy. Thus, it is important that the cells do not outgrow the plates, and that they are used as soon as possible. This is why they are cultured with neomycin for no longer than 30 days. These points are very important for the development of ES-cell-derived cellular therapies. The difficulties involved in freezing insulin-producing cells indicate that the cells must be cultured and used immediately for transplantation; there is no possibility of storage. The effect that trypsin seems to have on these cells also indicates that they can only stay in culture for a given period of time, until they outgrow the plates. Despite these drawbacks, our cell-trapping system based in the Nkx6.1 promoter results in a highly reproducible production of an appropriate number of insulin-containing cells that are in a relatively mature state in terms of glucose sensing and glucose-stimulated insulin release. Since our pioneering work [1], new strategies to induce in vitro differentiation have been developed. A common feature of these protocols is the formation of aggregates and addition of nicotinamide. Lumelsky et al. [20] used a cocktail of factors that expand nestin precursors. Methods based on this strategy result in a low insulin content and a high apoptotic rate, a situation compatible with insulin uptake from the culture media. An innovative method has been introduced by Blyszczuk et al. [21] driving differentiation of stem cells by constitutive expression of $\mathrm{Pax}-4$.

In this paper, we introduce new factors that separately promote differentiation. A combination of methods including sequential and/or combined exposure to factors known to promote differentiation may result in a strategy to generate islet cells from stem cells.

Acknowledgements. We thank F. X. Real for his support with this work. We also thank O. Madsen for providing the rabbit anti-Nkx6.1 antibody. This study was partially supported by grants from MCYT (Ministry of Science and Technology; GEN 2001-4748-C03-03, GEN 2001-4748-C05-05 and PM99412), Instituto de Salud Carlos III (G03/171, G03/212 and G03/210), the European Commission (QOL-2001-3), Fundación Progreso y Salud, Sevilla (Progress and Health Foundation, Seville), Fundació Marató TV3 (Marató Foundation of TV3), Generalitat de Catalunya (the region of Catalonia) and the European Foundation for the Study of Diabetes.

\section{References}

1. Shapiro AM, Lakey JR, Ryan EA et al. (2000) Islet transplantation in seven patients with type 1 diabetes mellitus using a glucocorticoid-free immunosuppressive regimen. New Engl J Med 343:230-238

2. Soria B, Roche E, Berná G, León-Quinto T, Reig JA, Martin F (2000) Insulin-secreting cells derived from embryonic stem cells normalize glycemia in streptozotocininduced diabetic mice. Diabetes 49:157-162

3. Soria B, Skoudy A, Martin F (2001) From stem cells to beta cells: new strategies in cell therapy of diabetes mellitus. Diabetologia 44:407-415

4. Perez-Villamil B, Schwartz PT, Vallejo M (1999) The pancreatic homeodomain transcription factor IDX1/IPF1 is expressed in neural cells during brain development. Endocrinology 40:3857-3860

5. Thomson J, Itskovitz-Eldor J, Shapiro SS et al. (1998) Embryonic stem cell lines derived from human blastocysts. Science 282:1145-1147

6. Watada H, Mirmira RG, Leung J, German MS (2000) Transcriptional and translational regulation of beta-cell differentiation factor Nkx6.1. J Biol Chem 275:34224-34230

7. Sander M, Sussel L, Conners J et al. (2000) Homeobox gene Nkx6.1 lies downstream of Nkx2.2 in the major pathway of beta-cell formation in the pancreas. Development 127:5533-5540

8. Oster A, Jensen J, Edlund H, Larsson LI (1998) Homeobox gene product Nkx6.1 immunoreactivity in nuclei of endocrine cells of rat and mouse stomach. J Histochem Cytochem 46:717-721

9. Schuldiner M, Yanuka O, Itskovitz-Eldor J, Melton D, Benvenitsy N (2000) From the cover: effects of eight growth factors on the differentiation of cells derived from 
human embryonic stem cells. Proc Natl Acad Sci USA 97:11307-11321

10. Otonkoski T, Beattie G, Mally M, Ricordi C, Hayek A (1993) Nicotinamide is a potent inducer of endocrine differentiation in cultured human fetal pancreatic cells. J Clin Invest 92:1459-1466

11. Chomczynski P, Sacchi N (1987) Single-step method for RNA isolation using guanidium thiocyanate-phenol-chloroform extraction. Anal Biochem 162:156-159

12. Mashima H, Ohnishi H, Wakabayashi K et al. (1996) Betacellulin and activin A coordinately convert amylase-secreting pancreatic AR42J cells into insulin-secreting cells. J Clin Invest 97:1647-1654

13. Krapp A, Knofler M, Ledermann B et al. (1998) The bHLH protein PTF1-p48 is essential for the formation of the exocrine and the correct spatial organization of the endocrine pancreas. Genes Dev 12:3752-3763

14. Huang HP, Liu M, El-Hodiri HM et al. (2000) Regulation of the pancreatic islet-specific gene BETA2 (neuroD) by neurogenin 3. Mol Cell Biol 20:3292-3330

15. Levinson-Dushnik M, Benvenisty N (1997) Involvement of hepatocyte nuclear factor 3 in endoderm differentiation of embryonic stem cells. Mol Cell Biol 17:3817-3822

16. De Bolos C, Garrido M, Real FX (1995) MUC6 apomucin shows a distinct normal tissue distribution that correlates with Lewis antigen expression in the human stomach. Gastroenterology 109:723-734

17. Rafic I, Kennedy HJ, Rutter GA (1998) Glucose-dependent translocation of insulin promoter factor-1 (IPF-1) between the nuclear periphery and the nucleoplasm of single MIN6 beta-cells. J Biol Chem 273:23241-23247

18. Skoudy A, Rovira M, Savatier P et al. (2004) Transforming growth factor (TGF) $\beta$, fibroblast growth factor (FGF) and retinoid signalling pathways promote pancreatic exocrine gene expression in mouse embryonic stem cells. Biochem J 379:749-756

19. Oster A, Jensen J, Serup P, Galante P, Madsen OD, Larsson LI (1998) Rat endocrine pancreatic development in relation to two homeobox gene products (Pdx-1 and Nkx6.1). J Histochem Cytochem 46:707-715

20. Lumelsky N, Blondel O, Laeng P, Velasco I, Ravin R, McKay R (2001) Differentiation of embryonic stem cells to insulin-secreting structures similar to pancreatic islets. Science 292:1389-1394

21. Blyszczuk P, Czyz J, Kania G et al. (2003) Expression of Pax4 in embryonic stem cells promotes differentiation of nestin-positive progenitors and insulin-producing cells. Proc Natl Acad Sci USA 100:998-1003 